\title{
Surface Generation Model in Grinding with Effect of Grain Shape and Cutting Speed*
}

\author{
Yoshifumi OHBUCHI** and Toshiyuki OBIKAWA***
}

\begin{abstract}
To simulate cutting with abrasive grain in grinding, a random grain shape is modeled. The statistical distribution of the effective rake angles of abrasive grain is obtained by applying Usui's four models of kinematically admissible velocity fields. The material is removed in the case of complete chip or incomplete chip formation, but is not removed when wall of partial chip or plastic upheaval is generated. Next, residual stock removal by bulging, which occurs due to effect of cutting speed, is presumed. A critical undeformed chip thickness, under which chip forms with great difficulty, is introduced. For further strict modeling, three components of cutting force are evaluated. As a result, a new model of surface generation process in grinding considering upheaval or residual stock removal, which is caused by the effect of grain shape and cutting speed, and of elastic deformation, is proposed.
\end{abstract}

Key Words: Grinding, Surface Generation, Grain Shape, Cutting Speed, Kinematically Admissible Velocity Field, Critical Undeformed Chip Thickness

\section{Introduction}

The fundamental process of grinding is microscopic cutting with each abrasive grain on the wheel periphery. In grinding, a huge number of three-dimensional micro cuttings are performed with different shape of grains, hence the chip formation mechanism is extremely complex. To clarify the phenomena, there are a large number of researches. Especially, single-grit cutting test ${ }^{(1)-(3)}$ or cutting with large negative rake ${ }^{(4)-(6)}$ were performed to simulate grinding chip formation processes. However, the shapes of cutting edge are irregular, and each chip formation is different and complicated. Also, there is several deformation state exist simultaneously, as cutting, ploughing, bulging etc.

The shapes of cutting edge are irregular because the grains are made from breaking of crystal, and they are very difficult to classify geometrically. However, it was clarified by experiment that the most of chip formation processes in actual grinding can be approximated as cutting

* Received 31st October, 2005 (No. 05-4239)

** Creative Engineering and Design Education Center, Kumamoto University, Kurokami, Kumamoto 860-8555, Japan. E-mail: yohbuchi@mech.kumamoto-u.ac.jp

*** Department of Mechanical and Control Engineering, Tokyo Institute of Technology, O-Okayama, Meguro-ku, Tokyo 152-8552, Japan.

E-mail: tobilawa@mes.titech.ac.jp with 1-3 rake faces ${ }^{(7)}$. In cutting with grain edge, material deformation modes can be classified depending upon the grain shape. One is chip formation. Another is generation of plastic upheaval or wall of partial chip, generally called as side flow. In this case, the material along the grain pass, which should become chip, is remained on work surface. On the other hand, to form chips or upheaval is subject to cutting conditions. Generally, chip is easily formed when grain depth of cut and cutting speed are large. In the case of small grain depth of cut or low cutting speed, chip formation does not occur, and the work surface integrity will be deteriorated ${ }^{(8),(9)}$. These two factors make prediction of surface integrity in grinding difficult.

In this research, a further realistic cutting model with abrasive grain is proposed, by introducing the effect of grain shape and cutting speed, for the simulation of surface generation in grinding.

\section{Calculation of Effective Rake Angle by Use of En- ergy Method}

\subsection{Simulation approach}

The grain apex is assumed to be triangular pyramid approximately, and normal directions of each face are distributed randomly. The direction of chip flow is decided with minimum energy.

Energy analysis method ${ }^{(7)}$ that Usui et al. had proposed is used for calculation of the direction of chip flow. This is a method based on the data of orthogonal cutting. 

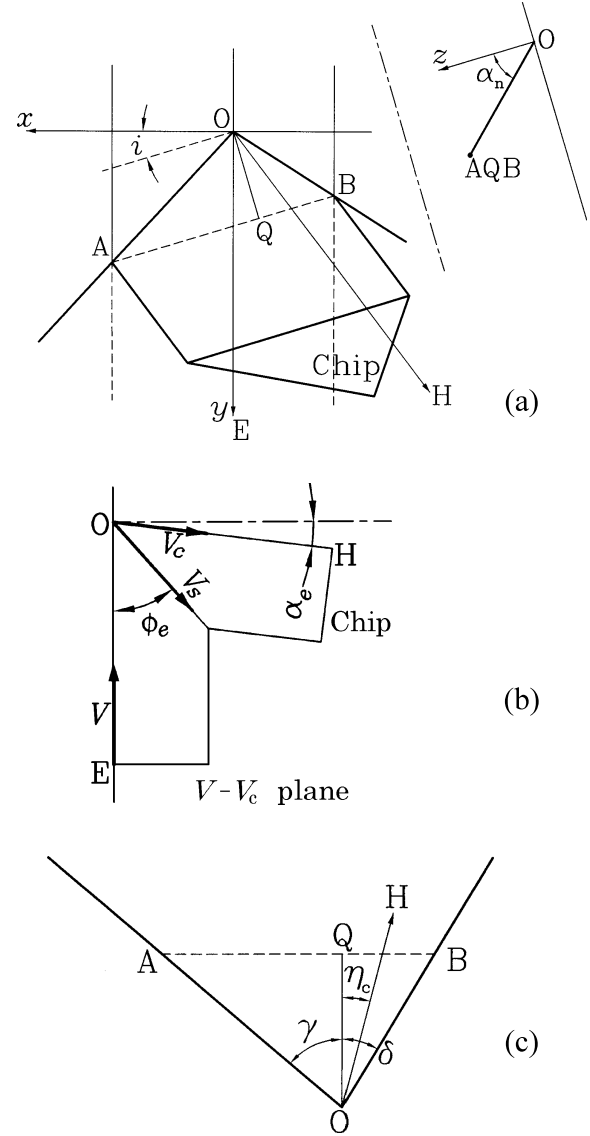

Fig. 1 Angle definitions concerning with triangular rake face

Under the assumption of kinematically admissible velocity field of three-dimensional cutting process, the deformation state is decided by searching of minimum energy. By means of this method, chip formation state and the cutting force components of three directions can be obtained just by calculation.

Applying to cutting process with abrasive grain, three-dimensional cutting with single triangular rake face on a grain is assumed, and the effective rake angle in the plane including cutting direction and chip flow direction

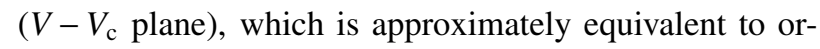
thogonal cutting, is determined. On the above-mentioned grain apex, it is assumed that the three-dimensional cutting is performed with 1 or 2 triangular rake faces. The chip flows to both sides independently on each rake face of the grain.

The angles concerning a triangular rake face are shown in Fig. 1. Though it differs from the definition of a usual cutting tool, according to Usui, normal rake angle $\alpha_{\mathrm{n}}$, effective rake $\alpha_{\mathrm{e}}$, inclination angle $i$, chip flow angle $\eta_{\mathrm{c}}$, cutting edge angle $\gamma$ and $\delta$ are defined. The rake face is triangle AOB. The $x-y$ plane is parallel to the work surface and it include grain apex $\mathrm{O}$. The $y$-axis is directed to cutting direction (Fig. 1 (a)). Here, the angle between rake face and $z$-axis is normal rake angle $\alpha_{\mathrm{n}}$ (it is shown as neg- ative angle in the figure). The angle of rotation around the $z$-axis of rake face is inclination angle $i$, and $\angle \mathrm{EOH}-\pi / 2$ in plane $\mathrm{EOH}$, which includes chip flow direction $\left(V-V_{\mathrm{c}}\right.$ plane), is effective rake angle $\alpha_{\mathrm{e}}$ as shown with shear angle $\phi_{\mathrm{e}}$ in Fig. 1 (b). Also, in Fig. 1 (c), which is seen from the normal direction of rake face, $\angle \mathrm{AOQ}$ and $\angle \mathrm{BOQ}$ are cutting edge angle $\gamma$ and $\delta$ respectively, and $\angle \mathrm{QOH}$ is chip flow angle $\eta_{\mathrm{c}}$.

As kinematically admissible velocity field, following four models Usui et al. proposed are used.

( 1 ) Model I: Complete chip formation.

( 2 ) Model II: Incomplete chip formation.

( 3 ) Model IV: Wall of partial chip generation.

( 4 ) Model V: Plastic upheaval generation.

Here, model III is omitted because it is not achieved energetically. By means of this energy method, model type, direction of chip flow and effective rake angle are determined.

Figure 2 shows the four models of three-dimensional chip formation by the kinematically admissible velocity field. In the case of model I, shown in Fig. 2 (a), a complete chip is formed. It is a state of the chip formation with negative rake angle in grinding process most generally imagined. When inclination angle of rake face becomes larger, unstable chip formation begins to occur. Then, incomplete chip of model II, shown in Fig. 2 (b), is formed. In this case, one side of edge does not take part directly in cutting, and the surface of chip becomes irregular shape. Next, wall of partial chip generation of model IV is appeared, when further increase of inclination angle occur. The wall of partial chip, which is partly generated to the side of cutting groove, is formed here, and the material remains on the work surface. Figure 2 (c) shows this state. In addition, when inclination angle increases more, deformation state of model V shown in Fig. 2 (d) occurs. Here, a low, wide stable upheaval wall is formed.

Energy method is based on experimental orthogonal cutting data. The relations between rake angle and shear angle, friction angle, yield shear stress on shear plane are necessary for the calculation. The orthogonal cutting experimental data for $0.50 \% \mathrm{C}$ carbon steel with changing rake angle form $-10^{\circ}$ to $30^{\circ}$, under the conditions of cutting speed of $2.5 \mathrm{~m} / \mathrm{s}$ and undeformed chip thickness of $0.205 \mathrm{~mm}$, is used for this calculation. Though extrapolation value from experiment of friction angle $\beta$ and shear angle $\phi_{\mathrm{e}}$ are corrected as next equation because an unreal geometrical solution was caused, when the experimental formula by Usui was enhanced to large negative rake angle.

$$
\begin{aligned}
& \beta=0.398+0.642 \alpha_{\mathrm{e}}+0.610 \exp \left(-\alpha_{\mathrm{e}}-1.571\right) \\
& \phi_{\mathrm{e}}=0.330+0.210 \alpha_{\mathrm{e}}+0.175 \exp \left(2.50 \alpha_{\mathrm{e}}-3.927\right)
\end{aligned}
$$

Here, these angles are in radian. Yield shear stress on shear plane $\tau_{\mathrm{s}}=588 \mathrm{MPa}$ is used because it does not 


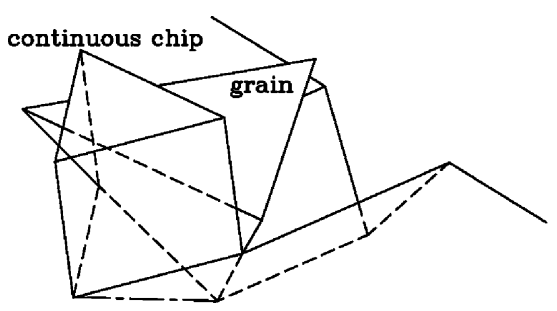

(a) Model I

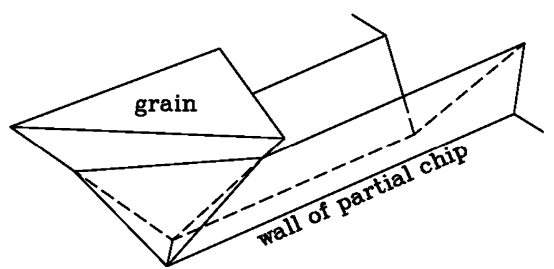

(c) Model IV

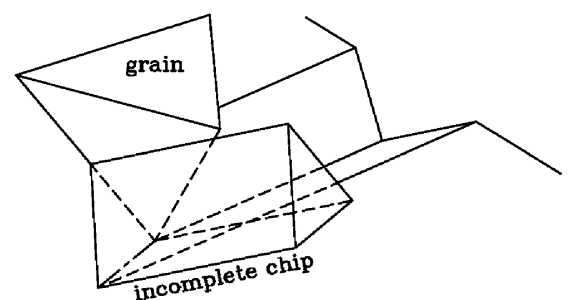

(b) Model II

Fig. 2 Four deformation models in cutting with grain by kinematically admissible velocity field, Usui et al. proposed

change with changing of rake angle in this orthogonal cutting experiment.

\subsection{Simulation results}

Figure 3 shows the result of effective rake angle distribution when normal rake angle $\alpha_{\mathrm{n}}$ is changed from $-5^{\circ}$ to $-85^{\circ}$, and inclination angle $i$ is changed from $0^{\circ}$ to $89^{\circ}$, and cutting edge angles $\gamma$ and $\delta$ are changed form $5^{\circ}$ to $85^{\circ}$ of uniform distribution by the above mentioned energy method. Here, the data with $\gamma+\delta<60^{\circ}$ are omitted because it seems to be unreal in actual grain shape. This distribution is obtained using 10 million calculated data.

From this figure, though normal rake angles given are all negative, effective rake angle calculated has wide distribution from negative value to positive value, and almost half have positive value. Model I with complete chip formation occur about $80 \%$, and most cases with effective rake angle of less than $30^{\circ}$ falls in this model. In the domain of effective rake angle larger than $30^{\circ}$, the percentages of model IV with wall of partial chip and model V with plastic upheaval increase, and it becomes predominant in the domain of effective rake angle larger than $65^{\circ}$. The parts of them may remain on the work surface, and deprave surface roughness.

The effective rake angle is larger than $-20^{\circ}\left(>-20^{\circ}\right)$, in the grain of majority. It belongs to the domain of ordinary cutting, in which well chip formation occurs. Remainder is cutting with large negative effective rake angle of $-20^{\circ}$ or less. In the current research of grinding, grain shape has been approximated as a conical shape with apex angle of from $120^{\circ}$ to $150^{\circ}$ degrees. Therefore, cutting process with abrasive grain has been thought to be cutting with rake angle of from $-60^{\circ}$ to $-75^{\circ}$. However, it is clear from this graph that such deformation occurs in low probability of about $5 \%$ or less. That is, the concept that grinding is accumulation of a huge number of cuttings
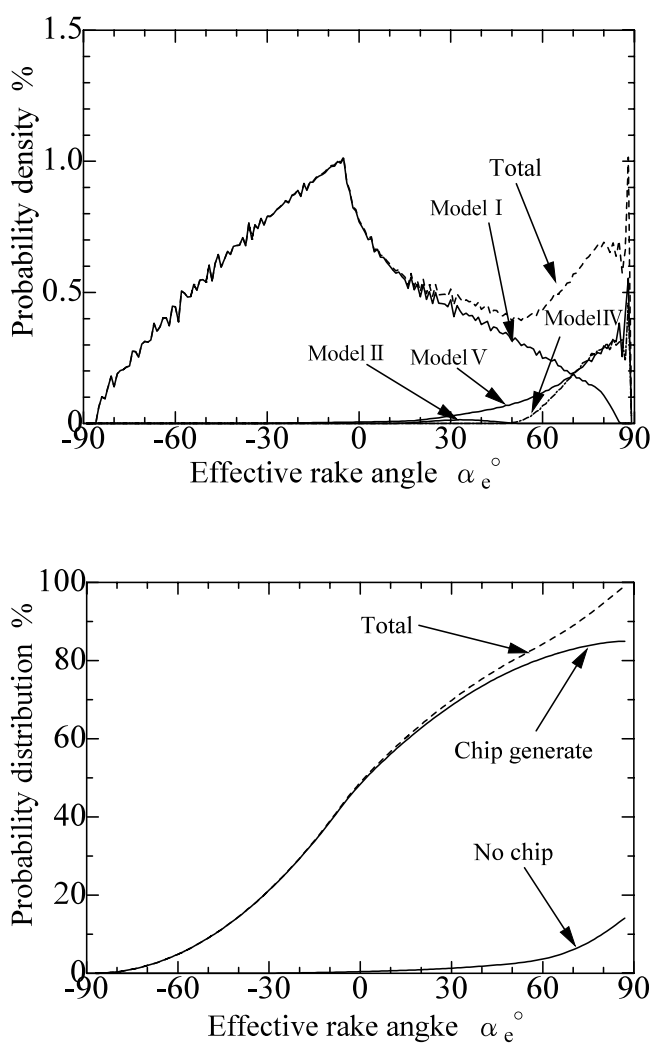

Fig. 3 Distribution of effective rake angle

with large negative rake angle is not necessarily correct.

\section{Relationship between Cutting Speed and Critical Undeformed Chip Thickness}

Next, the effects of cutting speed and undeformed chip thickness on the cutting phenomenon with large negative rake angle are considered. In the domain of large negative effective rake angle, multi deformation state with wall of partial chip generation of model IV or plastic up- 


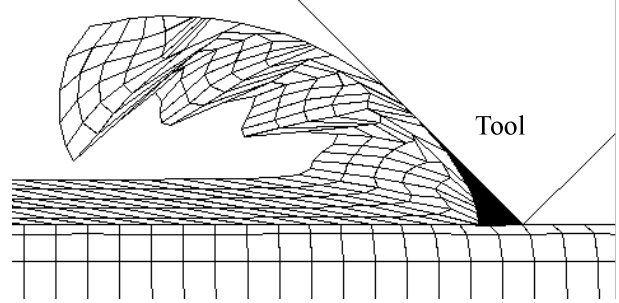

(a)

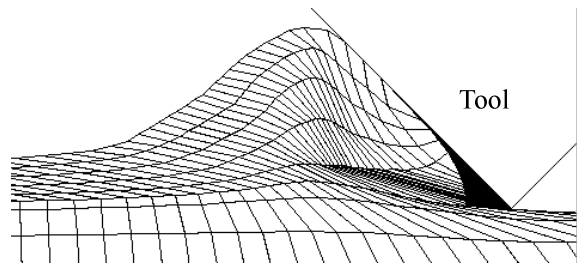

(b)

Fig. 4 Two typical results of finite element simulation. Cutting conditions; grain: WA, rake angle: $-45^{\circ}$, work: SK-5, cutting speed: $30 \mathrm{~m} / \mathrm{s}$, undeformed chip thickness: (a) $100 \mu \mathrm{m}$, (b) $1 \mu \mathrm{m}$

heaval of model $\mathrm{V}$ described above does not occur. Because only complete chip of model I is formed in cutting with negative effective rake angle as shown in Fig. 3, orthogonal cutting simulation with large negative rake angle proposed by authors ${ }^{(9)}$ can be applied.

Figure 4 shows the result of two typical finite element simulations. In (a), chip is well formed. On the other hand, in the condition of (b), the material is bulged ahead of rake face, but flow of chip to the upper side does not occur. In the case of low cutting speed or small undeformed chip thickness with large negative rake angle, bulging ahead of rake face is caused. However, this cannot be classified into above-mentioned wall of partial chip (model IV) or plastic upheaval to the grain side (model V) in large positive effective rake angle as shown in Fig. 3, because it is peculiar in cutting with large negative rake angle. This phenomenon depends on cutting speed and undeformed chip thickness, for the same effective rake angle. Therefore, the generation mechanism is fundamentally different. It is necessary to distinguish clearly. Actually, from this state of figure (b), it is thought to change into chip with ductile fracture or rupture, or to generate extremely large side flow without chip formation. In this paper, the state of (b), in which chip forms with great difficulty, is called as chip formation defective region. In cutting within this chip formation defective region, it is thought that deterioration of surface integrity will be caused by decrease in material removal efficiency, instability of deformation, and generation of the vibration etc.

According to the FEM simulation results, in which cutting speed and undeformed chip thickness are widely changed, there is a limit of undeformed chip thickness with stable chip formation. This limit decreases as cutting speed increases ${ }^{(9)}$. In Fig. 5, type of chip formation and critical cutting conditions on $V-h$ plane are shown. The critical undeformed chip thickness for stable chip formation decreases with increasing in cutting speed. Undeformed chip thickness of this boundary between stable chip formation region and chip formation defective region will be called as the critical undeformed chip thickness. Critical undeformed chip thickness indicates almost straight line on log-log scales. Because the slope is about

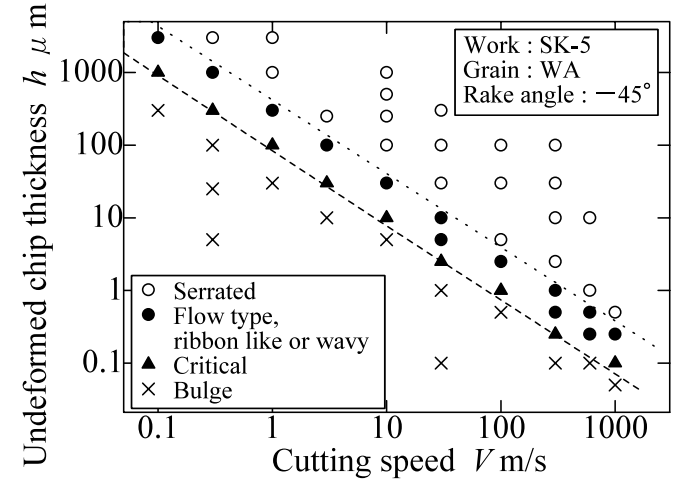

Fig. 5 Types of chip formation and the critical cutting conditions on $V-h$ plane for rake angle of $-45^{\circ}$

Table 1 Experimental conditions (Okoshi et al.)

\begin{tabular}{l|l}
\hline Grain shape & Triangular pyramid \\
Vertical angle & $120^{\circ}$ \\
Normal rake angle & $-45^{\circ}$ \\
Undeformed chip thickness & $20 \mu \mathrm{m}$ (maximum) \\
Cutting speed & 0 to $23.3 \mathrm{~m} / \mathrm{s}$ \\
\hline
\end{tabular}

-1 , the cutting speed is approximately inverse proportional to the critical undeformed chip thickness.

In order to verify accuracy of the simulation, obtained critical undeformed chip thickness should be confirmed with actual phenomenon. Okoshi et al. confirmed the grinding speed especially influences upheaval and residual stock removal remarkably by experiment ${ }^{(8)}$. It was forecast that this was closely related to the necessity of very high cutting speed in grinding compared with other machining. It seems that this phenomenon is related to the existence of critical undeformed chip thickness in this research. Then, critical undeformed chip thickness is compared with the experimental data of their upheaval and residual stock removal.

Okoshi's experimental conditions are shown in Table 1. In this experiment, undeformed chip thickness changes continuously from 0 to the maximum value along the width of cut, because $\mathrm{V}$ shaped groove had been created. That is, cutting under the same condition with a single tool, but only undeformed chip thickness was differ- 


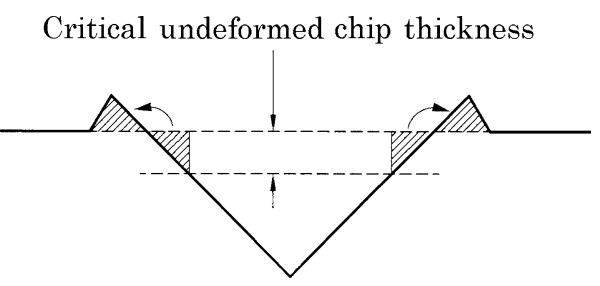

Fig. 6 Evaluated critical undeformed chip thickness by using upheaval or residual stock removal

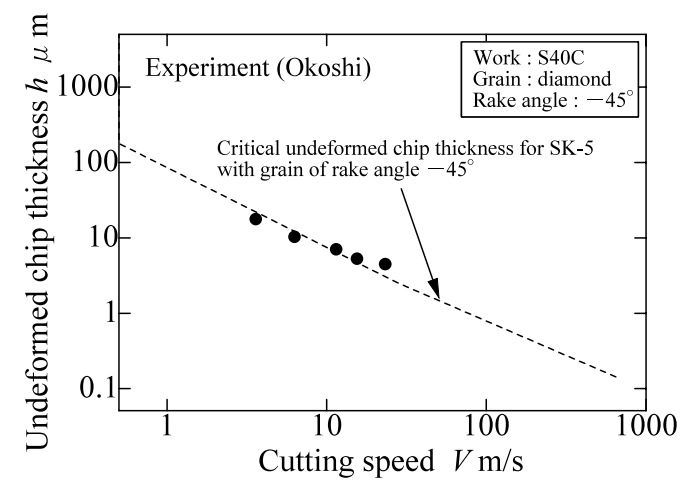

Fig. 7 Critical undeformed chip thickness calculated by FEM simulation and experimental data

ent, had been performed. Because the cutting was with symmetric tool geometry and its inclination angle was $0^{\circ}$, Usui's cutting model I is achieved in this state with chip flow angle of $0^{\circ}$. Considering the existence of critical undeformed chip thickness, chip may forms as undeformed chip thickness larger than the critical value. Side-flow occurs as the undeformed chip thickness smaller than the critical value.

Figure 6 shows the state of side flow in single grit cutting experiment. Assuming that all materials in the part of smaller undeformed chip thickness than critical value become side-flow, inversely it is clear from this figure that critical undeformed chip thickness is able to be calculated by using volume of side-flow. However, it cannot be calculated if maximum undeformed chip thickness is smaller than critical value. In this case, all material ahead of the grain becomes side-flow.

In Fig. 7, critical undeformed chip thickness calculated by above-mentioned procedure is shown, comparing with the result of FEM simulation. Though many kinds of materials are used in their experiments, the data of medium carbon steel S40C is used here, because it is thought to have close material properties with carbon tool steel SK-5 used in the FEM simulation. A good agreement of accuracy is shown in this figure. The feature that critical undeformed chip thickness decreases with increase in cutting speed is corresponding. Though the tendency for the single grit experimental data to exceed the value of the simulation slightly is seen at high cutting speed, influence of corner radius of shaped diamond is thought as a cause. As a result, existence of critical undeformed chip thickness, which had been shown by the FEM simulation, and effect of cutting speed on it were experimentally confirmed.

\section{Evaluation of Elastic Deformation}

For further strict modeling of surface generation with abrasive grain, the evaluation of cutting forces will become necessary. It is used for estimation of elastic deformation of grain, wheel, spindle and workpiece. However, though the cutting forces can be obtained by energy method, the effects of cutting speed and undeformed chip thickness are not considered in this model. Because in the orthogonal cutting experiment by Usui introduced here, cutting speed and undeformed chip thickness were constant. On the other hand, cutting forces are also obtained by FEM simulation. These cutting forces are based on the flow stress characteristic in which the effect of temperature, strain, and strain rate are considered ${ }^{(9)-(12)}$, and changes greatly at the critical undeformed chip thickness as shown in Fig. 8. But obtained forces here are for orthogonal cutting.

In order to obtain three components of cutting force in consideration of the effect of cutting speed and undeformed chip thickness, it is necessary to explain the parameters used in the energy method $\left(\beta, \phi_{\mathrm{e}}, \tau_{\mathrm{s}}\right)$ as functions of not only rake angle but also cutting speed and undeformed chip thickness. However, huge numbers of experiments are necessary, and it is very difficult to measure cutting forces and chip thickness in ordinary grinding. Hence, the following combination technique is proposed as an easier method to obtain forces in three-dimensional cutting.

(1) Effective rake angle and cutting forces (three components of force) are calculated for given grain shape by means of energy method.

(2) Specific cutting force in orthogonal cutting is calculated by FEM simulation, for effective rake angle obtained by energy method and given cutting speed.

(3) The ratio of two specific cutting forces, which are obtained by the FEM simulation and by energy method, is calculated.

( 4 ) Three components of force are evaluated by multiplying this ratio to three components of force obtained by energy method.

That means the cutting force influenced by cutting speed and undeformed chip thickness for the effective rake angle obtained by energy method can be calculated by means of FEM simulation, and the other two components (thrust force and lateral force) are calculated from the ratio of forces by two methods and result of energy method.

The result obtained is not sufficiently accurate, but good enough as comparing to using of orthogonal cutting parameters $\left(\beta, \phi_{\mathrm{e}}, \tau_{\mathrm{s}}\right)$ obtained by FEM. It includes the 


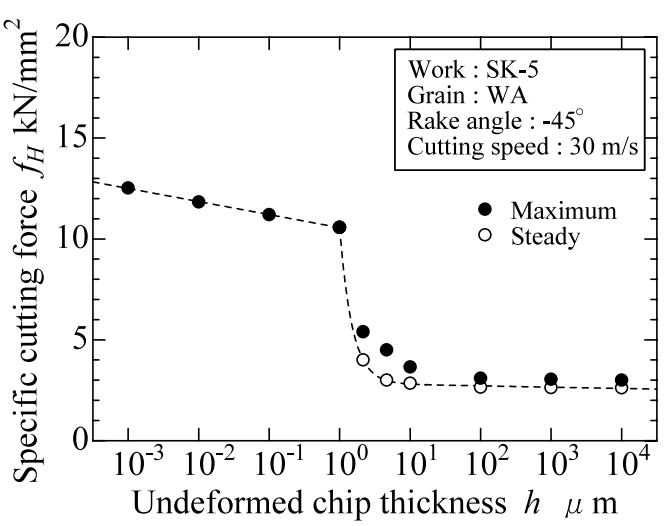

Fig. 8 Change of specific cutting force affected by undeformed chip thickness

effect of stagnant region ${ }^{(9),(13),(14)}$ and adiabatic shear ${ }^{(10)}$ caused in high-speed cutting with large negative rake angle. These deformations become different from the model Usui proposed. Though they consist of many planes of velocity discontinuity, searching minimum energy by energy method is not suitable, and the technique above mentioned is effective. In addition, for the more accurate result of thrust and lateral force with large negative rake angle, it is preferable to use $\beta$ and $\phi_{\mathrm{e}}$ obtained by FEM simulation instead of extrapolation value from experiment.

\section{Single Grit Cutting Model and Surface Genera- tion Model with Effect of Grain Shape and Cutting Speed}

Figure 9 shows the simulation result of changes of groove caused by effect of grain shape obtained by means of energy method. In the case of model I or model II, whole material in grain pass is completely removed as shown in (a), and it become complete or incomplete chip as described above. On the other hand, In the case of deformation of model IV or V, it is assumed that all materials move to direction of chip flow angle and it remains on work surface, and then, the profile after grain passes is provided as shown in (b) and (c). The wall of partial chip has sharp and large height as shown in (b), and surface roughness will become large.

Next, as effect of cutting speed, side-flow profile of equal sectional area to the part where is smaller than critical undeformed chip thickness, is generated at the both side of created groove. Figure 10 shows the simulated result of the effect of cutting speed. This is corresponding Okoshi's experimental data. Lower cutting speed causes large side-flow.

Figure 11 shows an example of calculated result using combination technique to simulate elastic deformation, described above. Orthogonal cutting experimental data used in energy method is for $0.50 \% \mathrm{C}$ carbon steel S50C, but flow stress characteristic of S50C with high reliability cannot be obtained, the data for $0.93 \% \mathrm{C}$ high car-

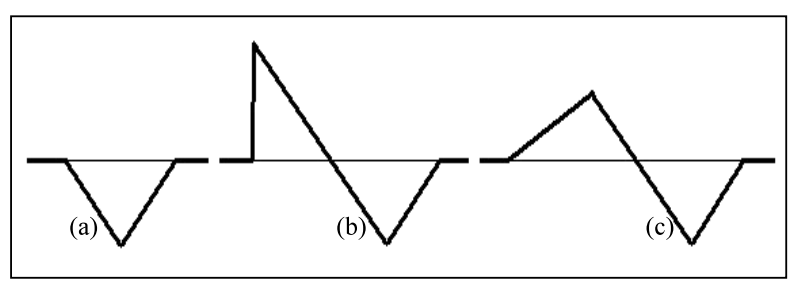

Fig. 9 Changes of grooves caused by effect of grain shape. (a) model I or model II, (b) wall of partial chip of model IV, (c) plastic upheaval of model V

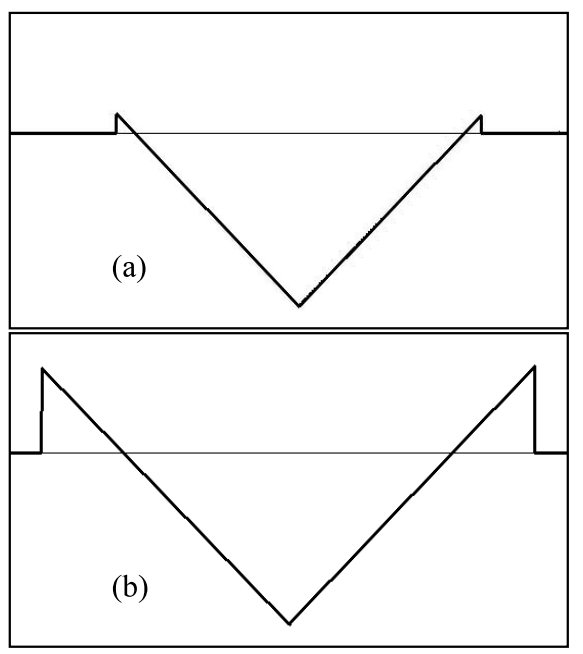

Fig. 10 Changes of grooves caused by effect of cutting speed. (a) $23 \mathrm{~m} / \mathrm{s}$, (b) $5 \mathrm{~m} / \mathrm{s}$. grain depth of cut: $20 \mu \mathrm{m}$

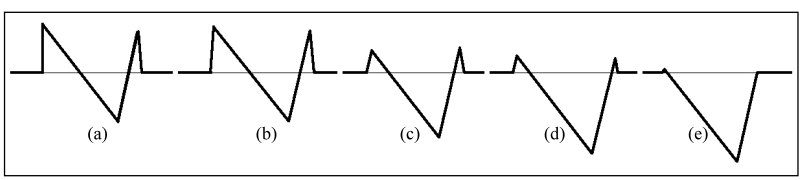

Fig. 11 Simulation result by combination method, Cutting conditions; grain depth of cut: $3 \mu \mathrm{m}$, cutting speed: (a) $10 \mathrm{~m} / \mathrm{s}$, (b) $50 \mathrm{~m} / \mathrm{s}$, (c) $100 \mathrm{~m} / \mathrm{s}$, (d) $150 \mathrm{~m} / \mathrm{s}$, (e) $300 \mathrm{~m} / \mathrm{s}$

bon tool steel SK-5 is used here. In addition, it is well known that specific cutting force increases with decrease of cross sectional area of cutting. This phenomenon is also introduced as given by $f_{\mathrm{c}}=\mathrm{Ca}_{\mathrm{m}}^{-\varepsilon}$, according to simulation by Sakai, et al. ${ }^{(15)}$ Here, $f_{\mathrm{c}}$ is specific cutting force, $a_{\mathrm{m}}$ is cross sectional area of cutting, and $C, \varepsilon$ are constant depending on material properties of work piece. In this figure, because supporting stiffness of grain is introduced, then, grain displacement with elastic deformation of wheel caused by thrust force is also simulated. The phenomenon that residual stock removal caused by grain displacement and plastic upheaval becomes small with increase of cutting speed is well simulated in this figure.

The surface generation result using all of models proposed above is shown in Fig. 12. Here, realistic generated surface is obtained and surface roughness by random shape grains and residual stock removal due to elastic de- 


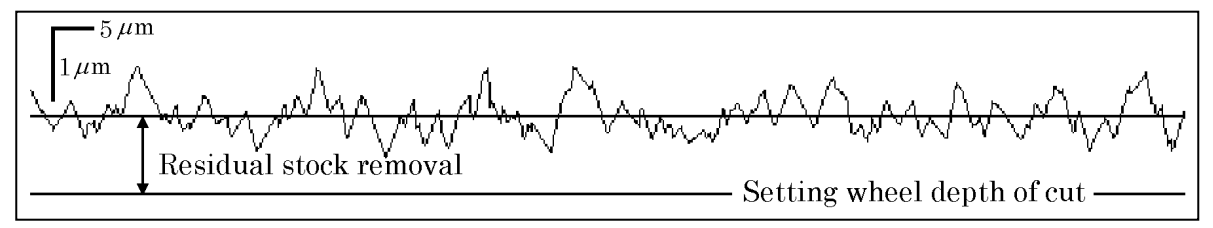

Fig. 12 Simulation result of surface generation, Cutting conditions; wheel: CBN140 N100 V, work: SK-5, wheel depth of cut: $10 \mu \mathrm{m}$, total depth of cut: $50 \mu \mathrm{m}$, spark out rotation: 5, cutting speed: $30 \mathrm{~m} / \mathrm{s}$, work speed: $10 \mathrm{~m} / \mathrm{min}$., spindle rigidity: $50 \mathrm{~N} / \mu \mathrm{m}$, work holding rigidity: $60 \mathrm{~N} / \mu \mathrm{m}$, grain contact rigidity: $0.4 \mathrm{~N} / \mu \mathrm{m}$

formation by grinding force are well simulated.

\section{Conclusions}

In this research, a new model of grain cutting in grinding was proposed. The grain apex was assumed to be triangular pyramid, and energy method was used together with the result of FEM simulation. The following results were clarified in consideration with effect of grain shape and cutting speed.

( 1 ) In three-dimensional cutting with abrasive grain with negative normal rake angle, probability density of large negative effective rake angle is less than that of rake angles of usual cutting region.

(2) The effect of cutting speed on critical undeformed chip thickness obtained by FEM simulation was corresponding to the single grit cutting experimental result.

(3) A new modeling of grain cutting was proposed. Here, consideration of upheaval or residual stock removal caused by the effect of grain shape and cutting speed, and effect of elastic deformation of grain supporting, were introduced. As results, generated surface profile and cutting forces can be calculated using Usui's energy method and FEM simulation data.

\section{References}

( 1 ) Rowe, G.W. and Wetton, A.G., Theoretical Considerations in the Grinding of Metals, Journal of the Institute of Metals, Vol.97 (1969), pp.193-200.

(2) Abebe, M. and Appl, F.C., Theoretical Analysis of the Basic Mechanics of Abrasive Processes (Part1), Wear, Vol.126 (1988), pp.251-266.

( 3 ) Matsuo, T., Toyoura, S., Oshima, E. and Ohbuchi, Y., Effect of Grain Shape on Cutting Force on Superabrasive Single-Grit Tests, Annals of the CIRP, Vol.38, No.1 (1989), pp.323-326.

( 4 ) Komanduri, R., Some Aspects of Machining with Negative Rake Tools Simulating Grinding, Int. J. Mach. Tool Des. Res., Vol.11 (1971), pp.223-233.
( 5 ) Malkin, S., Negative Rake Cutting to Simulate Chip Formation in Grinding, Annals of the CIRP, Vol.28, No.1 (1979), pp.209-212.

( 6 ) Ohbuchi, Y. and Matsuo, T., Force and Chip Formation in Single-Grit Orthogonal Cutting with Shaped CBN and Diamond Grains, Annals of the CIRP, Vol.40, No.1 (1991), pp.327-380.

( 7 ) Usui, E. and Inoue, M., Plasticity Analysis of Cutting Process with Abrasive Grain (1st Report), Journal of JSPE, (in Japanese), Vol.44, No.8 (1978), pp.988-994.

( 8 ) Okoshi, M., Yoshikawa, H. and Sata, T., Speed-Effect on Swell-Out Residual in Single Grain Grinding, Journal of JSPE, (in Japanese), Vol.25, No.10 (1959), pp.524-530.

(9) Ohbuchi, Y. and Obikawa, T., Finite Element Modeling of Chip Formation in the Domain of Negative Rake Angle Cutting, Journal of Engineering Materials and Technology, Transactions of the ASME, Vol.125 (2003), pp.324-332.

(10) Ohbuchi, Y. and Obikawa, T., Adiabatic Shear in Chip Formation with Negative Rake Angle, International Journal of Mechanical Sciences, Vol.47, Issue 9 (2005), pp.1377-1392.

(11) Yoshino, M. and Shirakashi, T., Flow-Stress Equation Including Effects of Strain-Rate and Temperature History, International Journal of Mechanical Sciences, Vol.39 (1997), pp.1345-1362.

(12) Horie, T., Finite Element Analysis of Mechanical and Thermal Damage in the Ground Surface Layer, Master Thesis, Tokyo Institute of Technology, (1993), pp.4446.

(13) Kita, Y., Ido, M. and Hata, S., The Mechanism of Metal Removal by an Abrasive Tool, Wear, Vol.47 (1978), pp.185-193.

(14) Kita, Y., Ido, M. and Kawasaki, N., A Study of Metal Flow Ahead of Tool Face with Large Negative Rake Angle, ASME Journal of Engineering for Industry, Vol.104 (1982), pp.319-325.

(15) Sakai, Y., Yagishita, H. and Furukawa, Y., Study on the Simulation of Grinding Process (1st Report), Journal of JSPE, (in Japanese), Vol.60, No.3 (1994), pp.367-371. 